\title{
Methods for absorbing kinetic energy with consideration of delay limit value
}

\author{
Jarostaw Rajczyk $^{1 *}$, Marlena Rajczyk $^{1}$, Jarosław Kalinowski ${ }^{1}$, and Budownictwa Wydział ${ }^{1}$ \\ ${ }^{1}$ Politechnika Czestochowska, 42-201 Czestochowa, Poland
}

\begin{abstract}
In this paper the potential of kinetic energy absorption by means of energy absorbing and storing mechanisms was discussed. The use of energy absorbers is intended to reduce the maximum working forces when stopping the body.
\end{abstract}

\section{Introduction}

The need to change speed or direction of movement occurs in many areas of life and technology, and this is particularly vital in transport. Incorporation of different speeds of vehicles, taking into account the collision intersection of their moving paths, necessitates speed changes. Another example is the formation of railway car drafts, where bumpers are used for connection and speed equalization is made mostly by braking. Another example is mooring a ship to the wharf where it contacts and slows down the ship's mass.

Braking processes are safe if speed dynamics are limited. Limiting dynamics limits the magnitude of the forces present in the braking process to safe values that do not cause permanent deformation of the braked objects or health hazard caused by mass forces affecting human organs.

In the case of standard braking of road or rail vehicles, the friction of the brake pads and the jaw is generally likely to produce heat that is released into the environment. In the case of traction vehicles, the so-called. Dynamic braking where the motors are switched to generator mode and the resulting energy is delivered to the traction network or lost on special heat resistance. This method does not cause wear on mechanical components. Similarly, hybrid cars are accumulating energy in batteries when braking, using it to drive the vehicle, reducing fuel consumption.

Utilizing even the most effective brakes does not allow for greater braking power in vehicles than the gravitational forces of the wheels to the road surface.

In the case of vehicular traffic, there are emergency situations of direct contact with an obstacle where the braking force values are not limited.

In the case of the formation of railway car drafts and mooring of ships, the intended physical contact of the objects must be reduced by dynamics to prevent their damage.

In particularly dangerous places where there are accidents caused by uncontrolled braking, for example, on racecourses, impact reduction devices are used. The aim of the study

*Corresponding author: jrajczyk@bud.pcz.czest.p1 
is to analyze the method useful in creating the structure of the absorber of kinetic energy resulting from the impact of mass on an obstacle $[7,8]$.

\section{Schemes of impact mechanisms}

In the event of a vehicle impact on the barrier, in order to stop the vehicle, the barrier must receive the kinetic energy of the vehicle. The kinetic energy of a vehicle is the sum of the energy associated with the relative speed of the vehicle to the obstacle and the kinetic energy of the rotation of wheels.

Energy intake should be controlled in such a way as not to exceed the maximum acceleration of driver safety.

In the case of traditional road barriers, the received energy is converted into a permanent deflection of the barrier and the barrier is destroyed. Spring barriers increase the braking force along with the deformation and, if not destroyed, cause the active braking of the braked vehicle, which is dangerous.

A natural non-destructive barrier to energy absorption in the case of body arrest is based on a spring part. Such methods are used in bumpers of railway cars where springs or polymeric parts are used. By using this method, the stopping process is spread over the length of the bumper deflection, and the collision energy of the cars is transmitted gradually causing the maximum impact forces to be reduced level. The energy stored in the resilient bumpers is returned, causing the cars to spread one from another. By spreading, the braking process over the bending deflections of the bumpers, the cars are not damaged by contact at higher speeds.

In the case of energy storage barriers, the energy transferred by the striking vehicle is converted into the energy stored in the barrier accumulator and is gradually reduced. There is no active force acting in the opposite direction when the vehicle decelerates. The energy absorbed by the accumulator will not be used to destroy it and will not be actively responded to. The barrier does not suffer any major damage and can be reused $[5,6]$.

\section{The collision with an inertial energy absorber in the form of free mass}

The scheme of the braking system is shown in Fig. 3. It assumes a system consisting of a vehicle with a mass of ms moving at the moment of initiation of braking at the initial velocity $V_{\text {pocz }}$, which acts through the sping part $R_{s}$ on the mass of the receiver $m_{0}$, which at its initial time is at rest $[4,9]$.

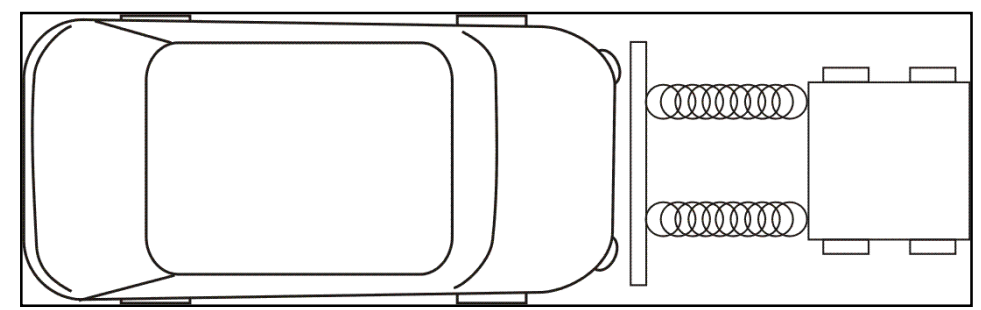

Fig. 1. Scheme of braking system using a spring and free mass.

The moving vehicle acts on the spring element to shorten it, causing the elastic force $F_{s}$ to counteract its shortening. The force that the elastic element interacts with the system is expressed in a linear equation:

$$
F_{s}=R_{s} s
$$


where $R_{s}$ - spring resilience in $\mathrm{N} / \mathrm{m}, S$ - shortening of spring in $\mathrm{m}$.

The force $F_{s}$ induced in the elastic element causes the vehicle to slow down and simultaneously increase the speed of the power receiver. The braking is terminated when the vehicle speed reaches zero or the spring reaches its initial length before the load.

Equations describing the behavior of the system in time $t$ take a form:

$$
\begin{aligned}
& F_{s}(t)=R_{s} s(t) \\
& V_{s}(t+d t)=V_{s}(t)+\frac{F_{s}}{m_{s}} d t \\
& V_{0}(t+d t)=V_{0}(t)-\frac{F_{s}}{m_{0}} d t \\
& S_{s}(t+d t)=S_{s}(t)+\frac{V_{s}(t+d t)+V_{s}(t)}{2} \\
& S_{0}(t+d t)=S_{0}(t)+\frac{V_{0}(t+d t)+V_{0}}{2} d t \\
& s(t)=S_{s}(t)-S_{0}(t)
\end{aligned}
$$

Equations describing the behavior of the system at the initial conditions $S_{0}(0)=0, V_{s}(0)$ $=V_{p}, V_{0}(0)=0$ and final condition $F_{s}>0$ for $t>0$.

where $R_{s}$ - elasticity of the elastic part, $F_{s}$ - force in elastic element, $V_{s}$ - car speed, $V_{p o c z}-$ the initial speed of the car, $S_{s}$ - the distance (position) of the car relative to its starting position at $t=0, m_{s}$ - mass of car, $V_{0}$ - energy receiver speed, $S_{0}$ - the distance (position) of the receiver relative to its initial position at time $t=0, m_{0}$ - mass of energy receiver.

In the analyzed system assuming the spring force of the vehicle with the mass of $m_{s}$ moving at the starting speed $V_{p s}$ with the fixed receiver $V_{p 0}=0$ with the mass $m_{0}$, the final velocity of the vehicle $V_{k s}$ and the receiver $V_{k 0}$ after the crash are described by the formulas:

$$
\begin{aligned}
V_{k s} & =V_{p s} \frac{m_{s}-m_{0}}{m_{s}+m_{0}} \\
V_{k 0} & =V_{p s} \frac{2 m_{s}}{m_{s}+m_{0}}
\end{aligned}
$$

There are distinctive behaviors of the system:

- $m_{s}>m_{0}-$ the vehicle will not stop completely $V_{k s}>0, V_{k 0}>0$,

- $m_{s}=m_{0}$ - the vehicle will stop completely $V_{k s}=0, V_{k 0}=V_{p s}$ (Exchange of speed and energy)

- $m_{s}<m_{0}$ - the vehicle will bounce off the receiver $V_{k s}<0, V_{k 0}>0$.

When the mass of the vehicle is equal to the mass of the receiver $m_{s}=m_{0}$, the speed change is complete, ie the car decelerates completely $V_{k s}=0$ and the speed of the receiver reaches the speed of the car before impact.

By substituting the formula of the ratio of the receiver to the car mass $m_{s} / m_{0}=c$ dependencies take the form:

$$
\begin{aligned}
& V_{k s}=V_{p s} \frac{1-c}{1+c}=V_{p s} d_{1} \\
& V_{k 0}=V_{p s} \frac{2}{1+c}=V_{p s} d_{2}
\end{aligned}
$$

The spring part should be selected so that the maximum allowable acceleration is not exceeded. The size of the device is proportional to the stopping distance which leads to its 
minimization. In case of optimum elasticity, the maximum acceleration value reaches a maximum value of $10 \mathrm{~g}$.

$$
\begin{aligned}
& d_{1}=\frac{1-c}{1+c}, \\
& d_{2}=\frac{2}{1+c} .
\end{aligned}
$$

The value $\mathrm{d} 1$ is a coefficient that determines the ratio of the vehicle's initial speed to its final speed. The $d_{2}$ determines the end speed of the receiver.

The graphs of $d_{1}$ and $d_{2}$, depending on the mass ratio between the receiver and the car masses, are shown in Figure 2.

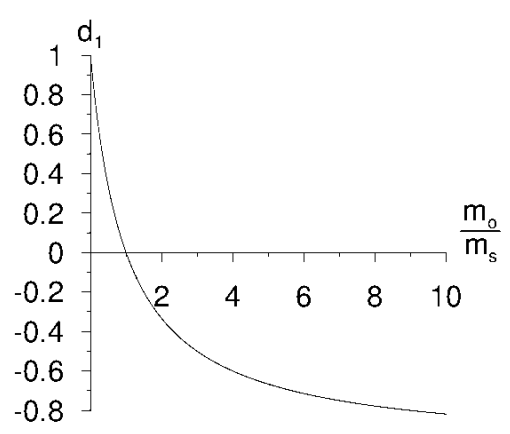

a)

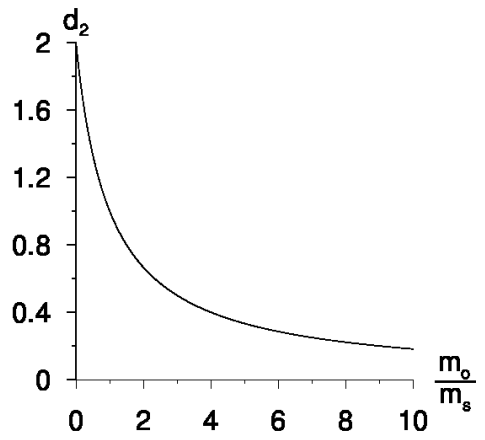

b)

Fig. 2. Diagrams of dependencies $d_{1}$ and $d_{2}$ between mass ratio of the receiver and the vehicle

The vehicle's final speed is determined by the product of $d_{1}$ and the initial velocity. The product of $d_{2}$ and the initial velocity of the vehicle determines the final speed of the receiver.

\section{Braking systems with rotating energy absorbers}

Under the inspiration of the EPAR project (energy dissipative crossover transmitter), a proposed solution to the kinetic energy receiver design from a moving vehicle consisting of the system is shown in Fig. 5

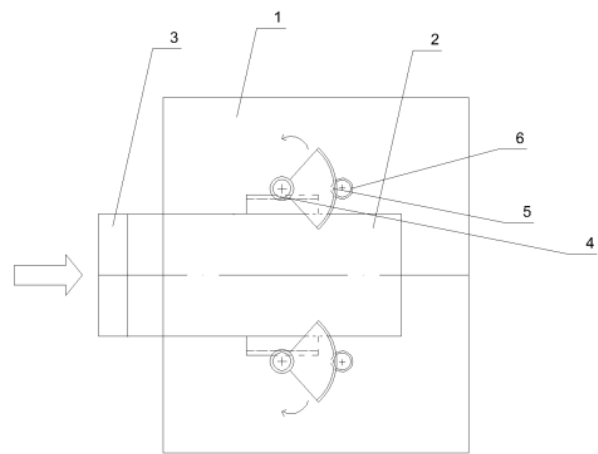

a) with a double kinetic energy accumulator [1]

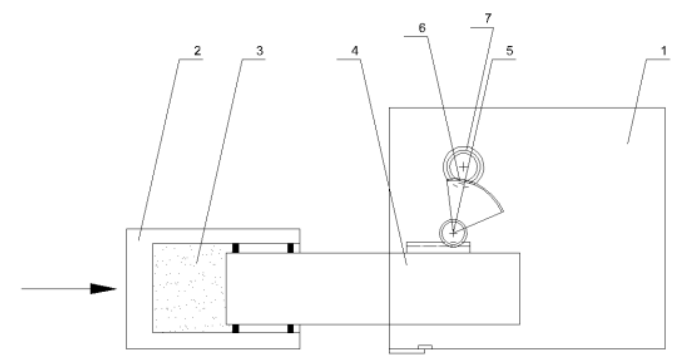

b) with a single kinetic energy accumulator [2]

Fig. 3. Conceptual diagrams of the EPAR system

The operation of the EPAR system in the event of the vehicle braking on an obstacle is to convert the kinetic energy of the vehicle's progressive motion into the energy of the rotary 
energy absorber in the vehicle or in the obstacle. The energy of the car is transmitted via a spring element to a linear gear that is part of a circular transmission [13, 14].

High gear ratio introduces the mass of the kinetic energy to the rotational motion. The device diagrams are shown in Figures $3 a$ and $3 b$.

Another solution is the construction of a road safety barrier by Rajczyk J., Rajczyk M., published in the Bulletin of the Patent Office, which is shown in Figure 4 [10].

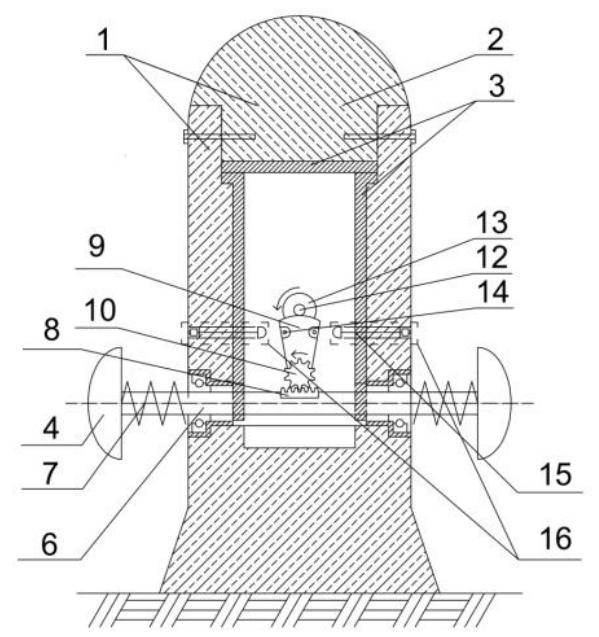

Fig. 4. The road barrier [3]

The road safety barrier consists a body (1) for absorbing kinetic energy, a top cover and a a stabilizing cover. The barrier kinetic energy absorbing assembly is formed of bumpers (4) arranged along opposite sides of the body together with the kinetic energy absorbing mechanisms attached to the body (1), each of which has a pivotal toothed arm (9) in the pivoting plane with ratchet- grippers (16) for their retention in the tilted position.

\section{Analysis of the braking process using a system of spinning spring energy absorber}

The system consists of a car moving at an initial speed that transmits its energy through the elastic parts to the insertion guide through a high pitched slide transmission made of a twisted flat bar with a corresponding spacer, hereinafter referred to as a worm gear, which introduces a rotatable absorber. The diagram of the example solution is shown in Fig. $5[11,12]$.

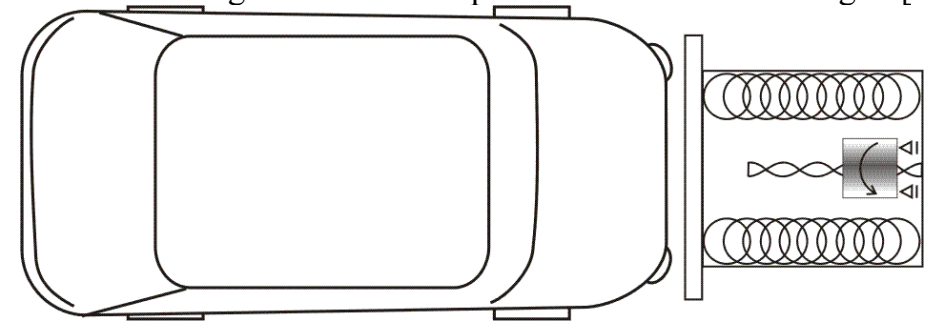

Fig. 5. Diagram of braking system spinning wheel with a worm gear, minimizing the size of the device.

The transmission has a pitch $\mathrm{f}$ at the offset for which the mass of the receiver is fully rotated. 
Regardless the friction forces in the transmission, the work done by the force $F$ to move the screw on the path $S$ corresponds to the work performed by the moment generated by the transmission at the angle of mass transfer of the receiver $\alpha$.

$$
F S=M \alpha .
$$

By substituting the above relationship for $S$, the linear pitch $\mathrm{f}$ and the full angle $\alpha$, we derive a relation determining the value of the torque acting on the receiver axis:

$$
M=\frac{F f}{2 \pi} .
$$

The value of acceleration is given by the formula:

$$
\varepsilon=\frac{F f}{2 \pi I} .
$$

The geometrical properties of the gearbox result in the relationship between the linear velocity of the worm gear $V_{z}$ and the angular velocity of the receiver expressed by the formula:

$$
V_{z}=\frac{f \omega}{2 \pi} .
$$

At the start of the braking process, the linear sprocket and the receiver are at rest. The worm gear reaches the velocity $V_{z}$ and makes the receiver reach angular velocity $\omega$ of formula 17.

The relationship between the linear acceleration $a_{z}$ and the angle $\varepsilon$ is given by the formula:

$$
a_{z}=\frac{f}{2 \pi} \varepsilon \text {. }
$$

The linear acceleration of the worm gear under the influence of the force acting on it is expressed by the formula:

$$
a_{z}=F \frac{f^{2}}{4 \pi^{2} I} .
$$

If instead of a rotary absorber, we use a linear energy receiver with a replacement mass $\mathrm{m}$, the acceleration of the absorber according to the second dynamic rule by the force $F$ would be:

$$
a_{z}=F \frac{1}{m_{z}} .
$$

Comparing the right side of the previous designs, the replacement mass of the linear absorber $\mathrm{m}$ with the corresponding rotary absorber was determined:

$$
m_{z}=\frac{4 \pi^{2} I}{f^{2}}
$$

The braking effect of the system is equivalent to that of a vehicle-spring -energy receiver for identical stiffness of the elastic parts and the substitute mass $m$, calculated according to the above formula 21. Comparison systems differ in the form of kinetic energy storage in respectively progressive and rotational motion.

Mass moment of inertia $I$ for a cylinder of radius $R$ and mass $\mathrm{m}$ is determined by: 


$$
I=\frac{1}{2} m R^{2}
$$

The radius of the cylindrical absorber of height $h$ from the material with material density $\gamma$ and the worm gear pitch $f$ corresponding to the replacement mass ms is given by the formula:

$$
R=\sqrt[4]{\frac{m_{s} f^{2}}{2 \pi^{3} h y}} .
$$

For example, for a vehicle with a mass of $m_{s}=900 \mathrm{~kg}$ moving at an initial $50 \mathrm{~km} / \mathrm{h}$, the optimal parameters of the rotary energy absorber with a worm gear pitch of $f=0.04 \mathrm{~m}$ were calculated for a cylinder at a height of $0.2 \mathrm{~m}$ made of concrete with a density of $\gamma=2500 \mathrm{~kg}$ $/ \mathrm{m}^{3}$.

The absorber radius for this system is $0.08255 \mathrm{~m}$ and its mass is $10.7 \mathrm{~kg}$. The calculated elasticity of the energy transferring part for the absorber for a maximum delay of $10 \mathrm{~g}$ was $R_{S}$ $=89.8596 \mathrm{kN} / \mathrm{m}$. For these parameters a numerical analysis of the system effect was performed, the results of which are presented in Fig. 6.
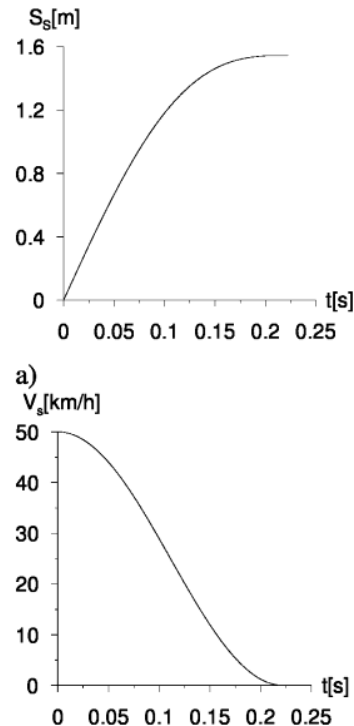

d)

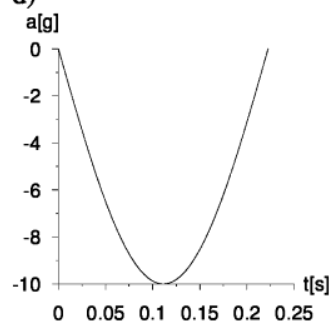

g)

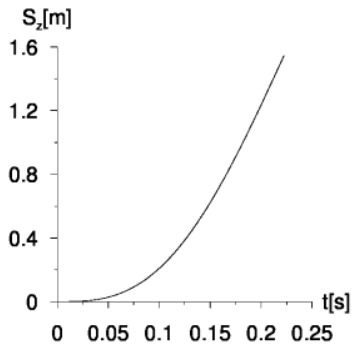

b)

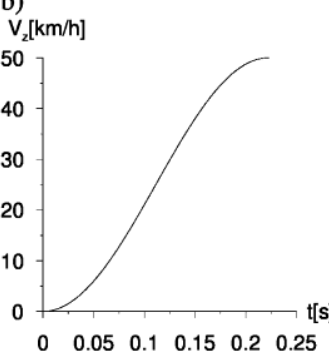

e)

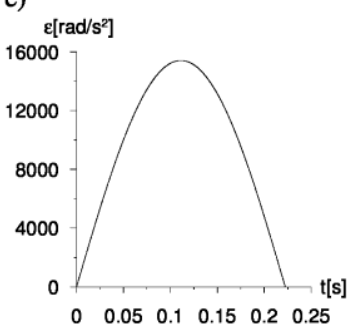

h)

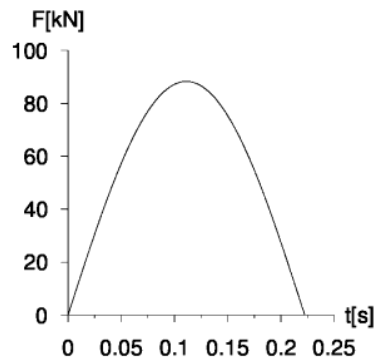

c)

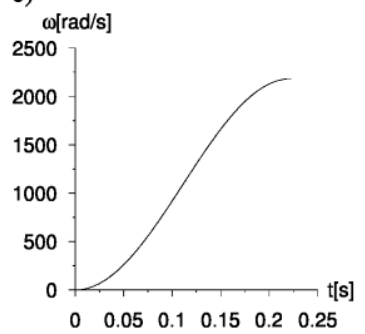

f)

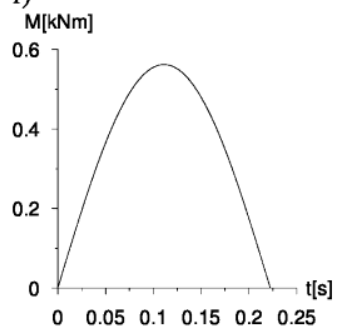

i)

Fig. 6. Here are the braking parameters specified in time for the energy of the rotating cylinder and) the road travelled by the vehicle, b) a linear displacement of the rack, c) the braking force acting on the vehicle, d) the vehicle speed, e) the linear velocity of the rack, f) the rotational velocity of the receiver $\mathrm{g}$ ) the delay value of the car, $\mathrm{h}$ ) the angular acceleration of the rotary power receiver, i) the torque acting on the receiver axis. 


\section{Conclusion}

In the study, considerations have been taken of the two vehicle braking systems - the compressed-mass of the receiver, the vehicle-spring part - rotary absorber and the equivalence of their braking performance. Due to the limitation of the space needed for the operation of the device, it is necessary to choose the rigidity of the spring parts and the mass or moment of mass inertia, depending on the considered system and the mass of the braked vehicle. Where the weight of the braked vehicle is less than that assumed in the calculation, the vehicle delay will be greater than the allowable value. At a greater mass than assumed, the delay will be less, which will increase the braking distance, and it will be necessary to use it to limit the displacement of the vehicle. Because the masses of vehicles are very different, it is not possible to build optimum universal collision protection based on these solutions. These devices will work best if they are adjusted to the weight of the braked vehicle. The ideal solution would be to install rotating energy absorbers in vehicles where they would be individually adjusted to their mass. The problem of vehicle reflection with incomplete transfer of energy to the receiver can be eliminated by the use of a ratchet mechanism that would permanently store energy.

Despite the construction difficulties, it is worth considering the use of a variable-speed worm gear, which would allow the elastic part to be skipped using the initial large worm gear pitch and the bumper may be redundant when using the self-locking gear at a small pitch. Proper selection of the variable screw travel length would allow for a uniform distribution of braking force.

\section{References}

1. S. Gumuła, L. Łągiewka, Conceptual design of vehicles protection against the impacts of collisions using the energy transfer method, Journal of KONES Powetrain and Transport, 13 (1), pp. 269-277 (2006)

2. S. Gumuła, L. Łągiewka A method of impact and intertia force reduction during collision between physical objects. Results of experimental investigations, Journal of Technical Physics, 48 (1), pp. 13-27 (2007)

3. Biuletyn Urzędu Patentowego RP Nr 21 (1090) str.27 (2015)

4. J. Rajczyk, E. Evtiukov, Dispersion of Kinetic energy for Traffic Safety. Transportation Research Procedia, 20, 536 - 543 (2017)

5. Liu Guowei, Xie Jun, Xie Suchao, Experimental and numerical investigations of a new $U$-shaped thin plate energy absorber subjected to bending and friction, Thin-Walled Structures, 115, pp. 215-224 (2017)

6. Gao Guangjun, Guan Weiyuan, Li Jian, Dong Haipeng, Zou Xiang, Chen Wei, Experimental investigation of an active-passive integration energy absorber for railway vehicles. Thin-Walled Structures, 117, pp. 89-97 (2017)

7. S. A. Evtiukov, Ya. V. Vasiliev, Reference book for RTA expert examination [Spravochnik po jekspertize DTP]. Saint Petersburg: Petropolis Publishing House, 512 p. (in Russian) (2015)

8. S. Evtiukov, G. Ginzburg, I. Brylev, Problem of accident reconstruction mechanism using parameters of braking process of twowheeled motor vehicles. Architecture and Engineering, (1)2, pp. 23-26. Available at: http://aej.spbgasu.ru/index.php/AE/issue/viewIssue/4/4 (viewed on 12.04.2016) (2016) 
9. J. Rajczyk, M. Rajczyk, J. Kalinowski, Methodology of the analysis of kinetic dispersion, Advanced Materials and Structural Engineering. ICA MEST-2015, Qingdao, China. (2015).

10. J. Rajczyk, P. Rajczyk, S. A. Evtiukov, Patent PL. 400082A1. Protective road barrier. (2014)

11. Sarli Matic, Turbulence kinetic energy. University of Lubliana. (2012)

12. James R Holton, An introduction to dynamic meteorology, Elsevier Academic Pres. touth edition. (2004)

13. Romanowski R. Wiktor Schanberger, genialny leśniczy. Projektowanie i konstrukcjie inżynierskie. XII. (2014).

14. F. T. Smith, Lifetime Matrix in Collision. Theory. Physical Review (1960). 